\title{
THE HIGH-RESOLUTION ELECTROSTATIC ENERGY ANALYZER FOR SPACE RESEARCH
}

\author{
Saulebekov A.O. ${ }^{1}$, Vénos D. ${ }^{2}$, Kambarova Zh.T. ${ }^{3}$ \\ 1 Lomonosov Moscow State University, Kazakhstan branch, Nur-Sultan, Kazakhstan \\ 2Nuclear Physics Institute, Czech Academy of Sciences, Řež, Czech Republic \\ 3Buketov Karaganda State University, Karaganda, Kazakhstan, kambarova@bk.ru
}

The energy analyzer of charged particle beams for space research is proposed. The developed electron-optical scheme of the energy analyzer of the charged particle beam is built on the basis of the electrostatic decapole-cylindrical field. The design of the energy analyzer is described. Equipotential portraits of the electrostatic decapole-cylindrical field are constructed. The trajectories of charged particles are calculated. A study of the parameters of the energy analyzer was carried out. The proposed energy analyzer has a high resolution and can be used in the development of spectrometers for space exploration.

Keywords: energy analyzer, electrostatic field, decapole-cylindrical mirror, electron-optical scheme, focusing order.

\section{Introduction}

Studying the dynamics of particle beams is of great interest for various problems that arise when constructing a theory of devices for studying the energy spectra of charged particles, the formation and transportation of charged particle beams in plasma and vacuum, and the injection of particle beams into plasma in laboratory conditions and in space.

The solution of problems related to highly sensitive analysis (substances in microelectronics, ecology, archeology, biology; geophysics, particle flows in interplanetary space in cosmic physics, etc.) was made possible thanks to the development and application of physical analysis methods. Moreover, today, the most widely used physical methods are spectroscopic methods, from which a group of actively developing corpuscular spectroscopy methods, including methods of electron spectroscopy, should be distinguished. However, there is a wide range of unresolved problems in this area, which determines the need for detailed theoretical and practical research. For example, the problems of modern spectral analysis are being actualized, which must be solved under conditions of a sharp increase in the requirements for resolution and sensitivity of the equipment, the complexity of the experiment geometry, the need to simultaneously investigation several parameters, speed up the research process, etc.

Space investigation is impossible to imagine without modern technology and equipment. Every year, for study extraterrestrial space, hundreds of satellites are launched into orbit, which are equipped with advanced equipment that allows to remotely study physical processes, their impact on the Earth, and record various space objects. Spectrometers, as the main tool for space investigation, began to be used more than half a century ago. In general, the modeling of spectrometers for research in space is an actual problem of science.

Cylindrical mirror type energy analyzers are widely used in the study of resonance phenomena in gases, in spectroscopy for chemical analysis, for obtaining spectra of secondary electrons, photoelectrons, autoelectrons, Auger electrons, as well as in space research, in studying the interaction of atomic particles with a solid surface and plasma diagnostics. 


\section{Axially-symmetrical multipoles}

For a potential multipole with plane symmetry $N$ in the cylindrical system coordinates, we can write the following [1]:

$$
U(r, \alpha, z)=\sum_{n=0}^{\infty} A_{n, N}(r, z) r^{n N} \cos n N \alpha
$$

The corresponding term $n=0$ describes the axially-symmetrical component of the field. Corresponding potentials for other members can be written as follows:

for $n=1$, this equation describes the distribution of the potential of an infinitely plane capacitor $U(x, y)=A_{1} x$; for $n=2$, this equation $U(x, y)=A_{2}\left(x^{2}-y^{2}\right)$ is this distribution of potentials is explained by the quadrupole, then are potentials of four alternating identical infinite hyperbolic surfaces. Accordingly, $n=3$ corresponds to a hexapole, $n=4$ to an octupole, $n=5$ to a decapole.

A mathematical method for constructing a new class of multipole-cylindrical fields formed by the superposition of a cylindrical field and circular multipoles was proposed in [2-3]. Based on the multipole approach, a wide range of various potential fields has been developed that are of practical interest for studying the mirror effect of these fields on a charged particles beam.

A wide variety of synthesized multipole-cylindrical fields opens up the prospect for the development of new effective energy analysis systems. For example, decapole-cylindrical fields, the potential properties of which have not yet been fully disclosed, may be of practical interest. It is necessary to continue theoretical studies that would allow to determine and expand the functionality of the practical application of multipole-cylindrical fields.

The electron-optical scheme of an electrostatic decapole-cylindrical mirror energy analyzer was firstly proposed in [4], in which the proportion of the cylindrical field and decapole are equal to $\mu=1$ and $\gamma=-1 / 100$ respectively. The trajectories of charged particles beam with the angular divergence in the axial plane were calculated. The electron-optical parameters of the proposed energy analyzer with the second-order angular focusing were calculated, and the optimal electronoptical scheme of device was determined.

The numerical modeling of the electron-optical scheme of the electrostatic decapole-cylindrical mirror energy analyzer at $\mu=1$ and $\gamma=-1 / 100$ was carried out in work [5]. The second-order angular focusing regimes of the "ring-ring" and "axis-ring" types were found. The focusing properties of the decapole-cylindrical energy analyzer were calculated.

The scheme of the energy analyzer based on the decapole-cylindrical field with the contributions of a cylindrical field $\mu=3 / 2$ and a circular decapole $\gamma=-1 / 100$ was proposed in [6]. Trajectory analysis of the motion of charged particles beams was performed. The design features of the scheme of the proposed energy analyzer were described. Numerical models of energy analyzer schemes based for two angular focusing regimes are obtained.

In this work, the high-resolution energy analyzer based on the decapole-cylindrical field for space research was proposed.

\section{Scheme of decapole-cylindrical mirror energy analyzer}

The potential of the deflecting field is a superposition of a cylindrical field and a circular decapole

$$
U(\rho, z)=\mu \ln (1+\rho)+\gamma U_{d}(\rho, z)
$$


where $\ln (1+\rho)$ is cylindrical field, $\mu$ is coefficient specifying the weight contribution of the cylindrical field, $U_{d}(\rho, z)=U_{0} \rho\left(z^{4}-2 \rho^{2} z^{2}+\frac{1}{5} \rho^{4}\right)$ is circular decapole, $\quad \gamma \quad$ is weight contribution of circular decapole.

The algorithm for determining equipotential lines in a decapole-cylindrical field is presented below.

$$
\begin{aligned}
& U_{d}(r, Z)=U_{0}\left[\rho\left(\xi^{4}-2 \rho^{2} \xi^{2}+\frac{1}{5} \rho^{4}\right)\right]=U_{0} f_{d}, \\
& U=U_{0}\left[\mu \ln r+f_{d}\right], \\
& \frac{U}{U_{0}}=\mu \ln r+f_{d}, \\
& -\frac{U}{U_{0}}+\mu \ln (1+\rho)+\rho\left(\xi^{4}-2 \rho^{2} \xi^{2}+\frac{1}{5} \rho^{4}\right)=0, \\
& \text { 1) } \frac{U}{U_{0}}=0 ; 0.1 ; 0.2 ; \ldots 1 .
\end{aligned}
$$

2) We set the values of $\rho$ from 0 ..... 1;

3) We obtain the values of $\xi$ for $R(\xi)=0$;

$$
R(\xi)=\frac{-U}{U_{0}}+\mu \ln (1+\rho)+\rho\left(\xi^{4}-2 \rho^{2} \xi^{2}+\frac{\rho^{4}}{5}\right)
$$

Given

$$
R(\xi)=0
$$

Find $(\xi) \rightarrow$

\section{Results}

The fig.1 (a-g) presents families of equipotential portraits of an electrostatic decapolecylindrical field with various contributions of a cylindrical field $\mu$ and a circular decapole $\gamma$. The analyzer field can be constructed in the form of a superposition of the base field and a set of circular multipoles, which coaxially with the base field.

Due to the small component of the circular field, the distribution of equipotentials of the decapole-cylindrical field, which have a slight deviation from straight lines, is close to the distribution of equipotentials of the cylindrical field.

The energy analyzer consists of an inner cylindrical electrode which is at zero potential, and axially symmetrical outer deflecting electrode, which under potential and the generatrix of which coincides with one of the equipotentials of the decapole-cylindrical field.

The scheme of the energy analyzer with a decapole-cylindrical field (2), in which the contributions of the cylindrical field and circular decapole are equal to $\mu=1$ and $\gamma=-\frac{1}{100}$ respectively, is shown in Fig. 2. According to the scheme, a beam of charged particles emerging from the ring source $\mathbf{A}$ is reflected by the mirror field and focused into the ring image $\mathbf{B}$.

The trajectories of charged particles motion were calculated by using the approximate analytical method for calculation the trajectories of charged particles in multipole-cylindrical fields. Results of calculation the axial trajectory in a decapole-cylindrical field are presented in table 1. 


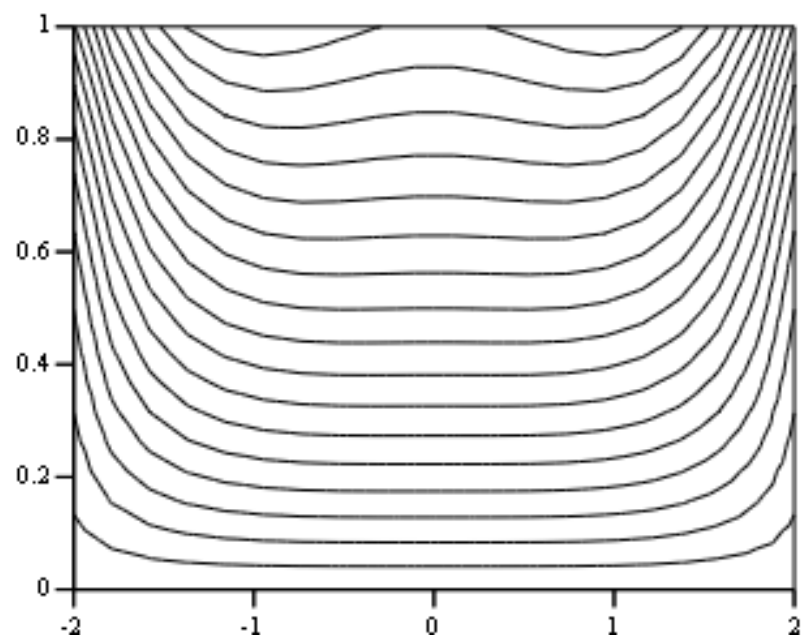

CreateMesh $(\mathrm{R},-2,2,0,1)$

a) $\mu=2.5, \gamma=-0.1$

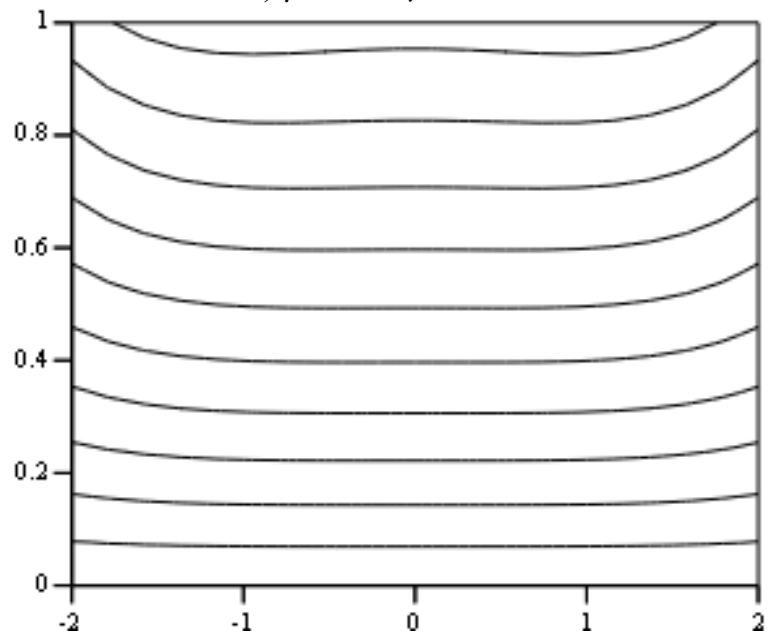

CreateMesh $(\mathrm{R},-2,2,0,1)$

c) $\mu=1.5, \gamma=-0.01$

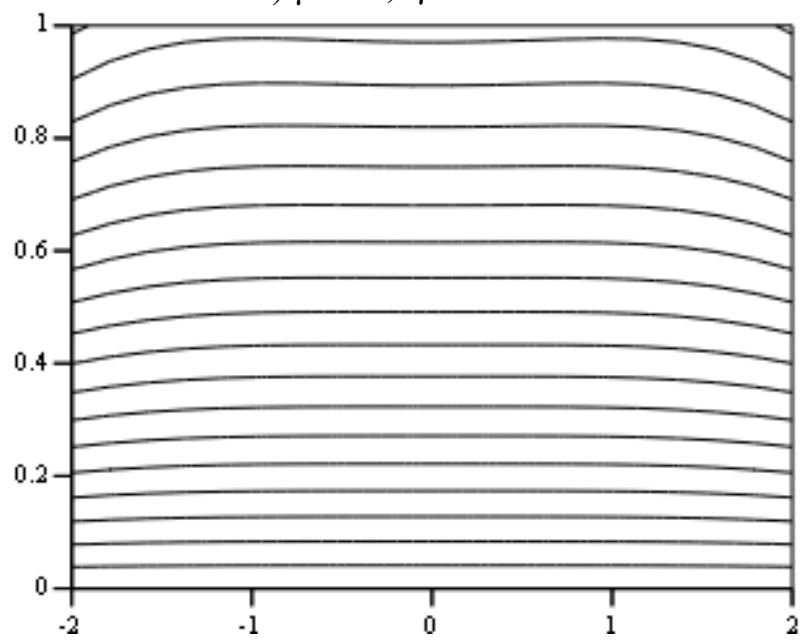

CreateMesh $(\mathrm{R},-2,2,0,1)$

f) $\mu=2, \quad \gamma=0.01$

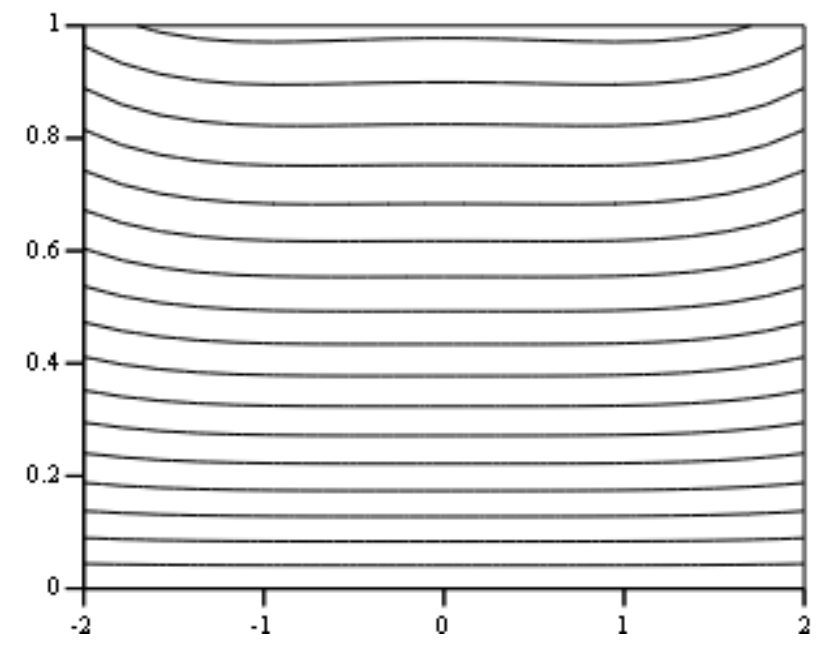

CreateMesh (R, -2, 2, 0, 1)

b) $\mu=2.5, \gamma=-0.01$

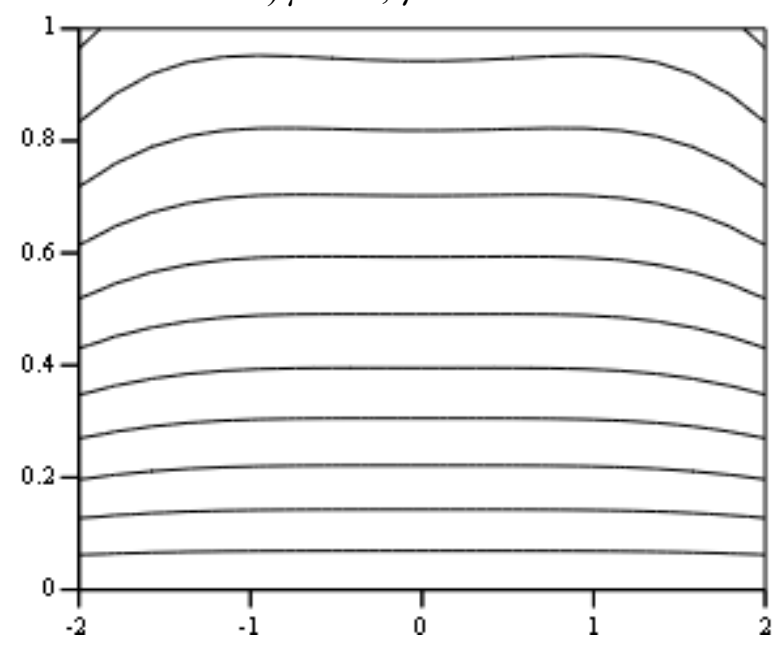

CreateMesh $(\mathrm{R},-2,2,0,1)$

d) $\mu=1.5, \gamma=0.01$

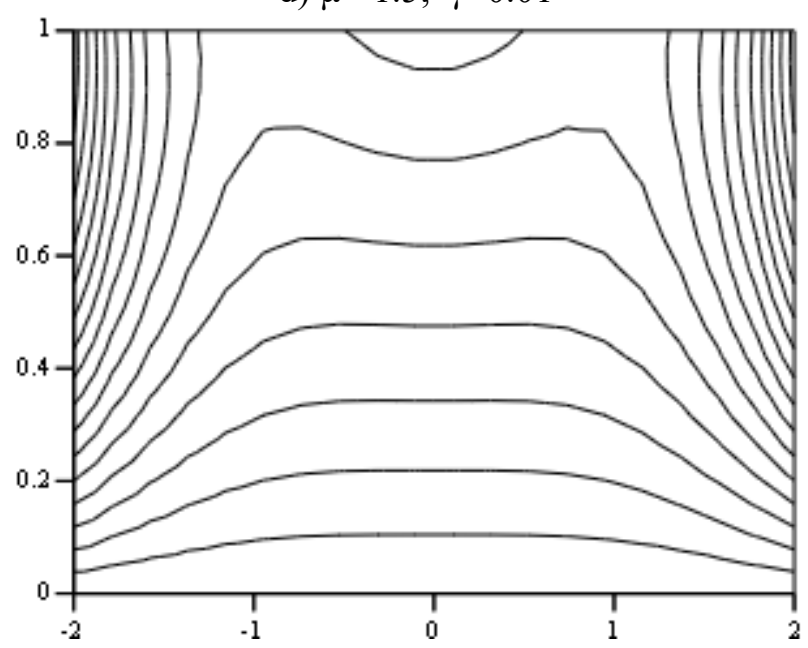

CreateMesh $(\mathrm{R},-2,2,0,1)$

g) $\mu=1, \gamma=0.1$

Fig. 1. Families of equipotential portraits of the electrostatic decapole-cylindrical field with various contributions of a cylindrical field and a circular decapole 


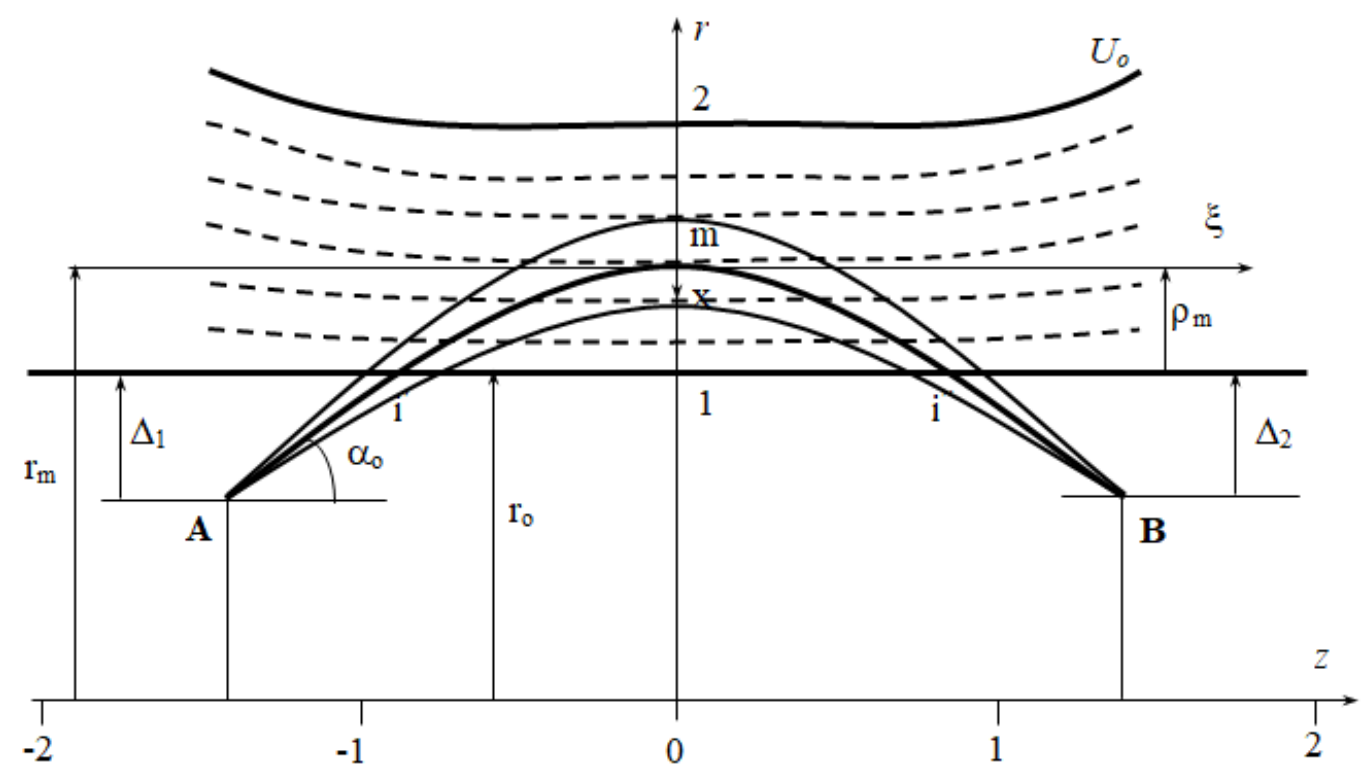

A is the source of charged particles, $i^{\prime}$ is entrance ring slit, $i^{\prime \prime}$ is exit ring slit, $\mathrm{B}$ is the detector

Fig. 2. The scheme of the energy analyzer based on the decapole-cylindrical field, in which the shares of the cylindrical field and decapole are equal to $\mu=1$ and $\gamma=-1 / 100$ respectively.

Table 1 -Results of calculation the axial trajectory in a decapole-cylindrical field

\begin{tabular}{|c|c|c|c|c|c|c|c|}
\hline$P$ & $\begin{array}{c}\alpha_{o}, \\
\text { degree })\end{array}$ & $R=1+\rho_{m}$ & $\xi_{m}$ & $\Delta$ & $l$ & $D$ & $A_{\text {III }}$ \\
\hline 0.5500 & 36.8346 & 1.3543 & 0.9896 & 0.3968 & 3.0386 & 2.3834 & -8.438 \\
\hline
\end{tabular}

where $\alpha_{o}$ is the entrance angle of the trajectory; $\Delta=\Delta_{1}=\Delta_{2}$ is the amount of removal of the source and its image from the surface of the inner cylindrical electrode; $\xi_{m}$ is the half projection of the trajectory onto the symmetry axis in the field of a decapole-cylindrical mirror, $R=1+\rho_{m}$ is the coordinate of the turning point of the trajectory, $l$ is the focal length equal to the full projection of the trajectory onto the symmetry axis of the mirror from the source to its image, satisfying the focusing conditions, $D=\frac{\partial l}{\partial \varepsilon}$ is the relative linear dispersion in energy, $A_{I I I}=\frac{1}{3 !} \frac{d^{3} l}{d \alpha^{3}}$ is coefficient of cubic angular aberration. All longitudinal parameters are expressed in units of the radius $r_{0}$ of the inner cylindrical electrode.

Using numerical calculation by the R-Kutta method, the following data for the lateral branches of trajectory are obtained (table 2). The initial angular spread is $12^{\circ}, \Delta \alpha=\alpha-\alpha_{o}= \pm 6$.

Table 2 -Results of calculation the lateral branches of trajectory

\begin{tabular}{|c|c|c|c|c|}
\hline$l(0)$ & $l(-6)$ & $l(+6)$ & $\Delta \mathrm{l}( \pm 6)$ & $\delta=\frac{D}{\Delta l}$ \\
\hline 3.0478 & 3.0526 & 3.0506 & 0.0076 & 313.6 \\
\hline
\end{tabular}


Thus, the specific energy dispersion, which characterizes the resolution of the decapolecylindrical field, calculated for particles with an initial angular spread of $12^{\circ}$, is twice the specific dispersion of the cylindrical mirror analyzer $\Delta L( \pm 6)=0.036$ [7].

Fig. 3 shows the dependences of the particle arrival point on the entrance angle $\alpha_{0}$ for the angular focusing regime of the "ring-ring" type for various values of the energy of the charged particles. The curve (for a particle with energy $E / V=1$ ) corresponds to the second-order angular focusing. It can be seen from Fig. 3 that the optimal range of entrance angles of particles to analyzer field is the range of angles from $30^{\circ}-42^{\circ}$, providing a maximum luminosity $\Omega=12 \%$ and the best focusing of the particle beam.

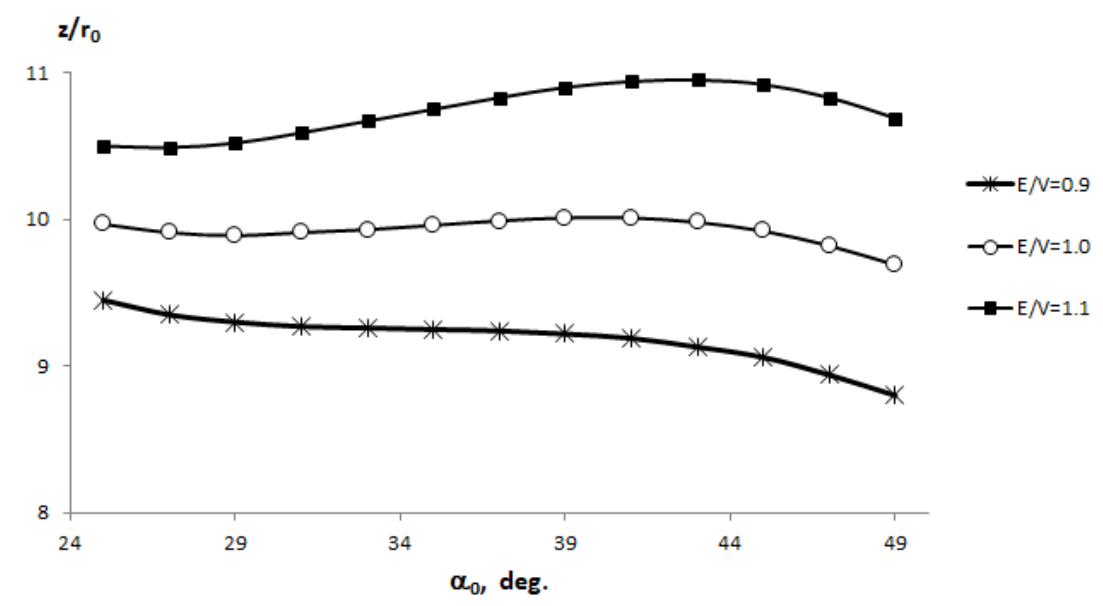

Fig. 3. The dependence of the particle arrival point on the entrance angle $\alpha_{o}$

\section{Conclusion}

The model of the device will make it possible to register flows of elementary particles from cosmic space, to obtain various spectra with high resolution. The device is compact and lightweight, and can be installed in a spacecraft. The proposed device can be used in various intermediate stages of matching particle beams, for example, for studying ion fluxes in cosmic space, for studying the elemental composition of celestial bodies in space research.

\section{REFERENCES}

1 Silad'i M. Electron and ion optics. Moscow: Mir, 1990, - 639 p.

2 ZashkvaraV.V., Tyndyk N.N. Axial-symmetrical multipoles, their application.Zh.Tech.Phys., 1991, Vol.61, No.4. pp.148-157.

3 Zashkvara V.V., Tyndyk N.N. Potential fields based on circular multipole series. Nuclear Instrum. and Meth., 1996, Vol. A370, pp.452-460.

4 Ashimbayeva B.U., Chokin K.Sh., Saulebekov A.O., Kambarova Zh.T.Decapole-cylindrical mirror energy analyzer. Bulletin of Karaganda University, 2012, No. 4 (68), - pp. 86-92.

5 Kambarova Z.T., Trubitsyn A.A., Saulebekov A.O. Axially symmetric energy analyzer based on the electrostatic decapole-cylindrical field. Technical Physics, 2018, Vol.63(11), pp.1667-1671. doi.org/10.1134/S1063784218110142

6 Kambarova Z.T., Saulebekov A.O., Saulebekova D.A. Modeling of electrostatic decapolecylindrical mirror analyzer. IOP Conference Series: Materials Science and Engineering 12, 2017. pp. 012078. DOI:10.1088/1757-899X/168/1/012078

7 Zashkvara V.V., Korsunskiy M.I., Kosmachev O.S. Focusing properties of an electrostatic mirror with a cylindrical field. Zh.Tech.Phys., 1966, Vol.36, No.1, pp. 132-138. 